\title{
Clinical characteristics, gestational weight gain and pregnancy outcomes in women with a history of gestational diabetes mellitus
}

\author{
Xin Liang, Wei Zheng, Cheng Liu, Lirui Zhang, Li Zhang, Zhihong Tian and Guanghui Li
}

\begin{abstract}
Background: Pregnant women with a history of gestational diabetes mellitus (GDM) are at high risk of GDM. It is unclear whether this population has pregnancy characteristics different from the general population. Whether these features affect the perinatal outcome has not yet been elucidated.

Methods: A retrospective study was conducted, including baseline characteristics, laboratory data, gestational weight gain (GWG), and pregnancy outcomes of 441 pregnant women with prior GDM. Besides, 1637 women without a history of GDM treated in the same period were randomly selected as the control group. The above indicators of the two groups were compared. Multivariable logistic regression analysis was performed to investigate how GWG was associated with perinatal outcomes for previous GDM women.
\end{abstract}

Results: Among women with GDM history, triglycerides (TG) and fasting plasma glucose (FPG) in the 1st trimester were higher than those without GDM history. GWG was lower in women with prior GDM relative to the control group at various pregnancy stages. However, women with GDM history had a higher risk of developing GDM (OR 3.25, 95\% Cl 2.26-4.68) and pregnancy-induced hypertension (OR 1.50, 95\% Cl 1.05-2.45). In women with previous GDM, excessive GWG before OGTT exhibited a positive correlation with pregnancy-induced hypertension (OR 1.47, 95\% Cl 1.05-3.32), while inadequate GWG was not a protective factor for GDM and pregnancy-induced hypertension.

Conclusion: Women with prior GDM have glucose and lipid metabolism disorders in the 1st trimester. Limited reduction of GWG before oral glucose tolerance test (OGTT) was insufficient to offset the adverse effects of glucose and lipid metabolism disorders in women with previous GDM. Relevant interventions may be required at early stage or even before pregnancy.

Keywords: A history of gestational diabetes mellitus, Glucose and lipid profiles, Gestational weight gain, Pregnancy outcomes

\section{Background}

Gestational diabetes mellitus (GDM) is a prevalent pregnancy metabolic disorder characterized by insulin resistance and impaired pancreatic $\beta$-cell function $[1,2]$. It can lead to adverse perinatal outcomes and influence

*Correspondence: liguanghui@ccmu.edu.cn

Division of Endocrinology and Metabolism, Department of Obstetrics, Beijing Obstetrics and Gynecology Hospital, Capital Medical University, No 251, Yaojiayuan Road, Chaoyang District, Beijing 100026, China long-term health of mothers and their offspring [3]. In recent years, the incidence of GDM in CHINA has reached 17.5-18.9\% [4]. The promotion of "two-child" policy in China has recently significantly increased the rates of high-risk pregnancy and adverse pregnancy outcomes $[5,6]$. Particularly, the number of pregnant women with GDM history has increased, and previous studies suggest that GDM history is a risk factor for the recurrence of GDM and type 2 diabetes [7, 8]. It was reported that women with prior GDM had a tenfold increased 
risk for type 2 diabetes compared to those without GDM history [9]. There was a higher likelihood of developing type 2 diabetes in women who were overweight or obese before pregnancy with a history of GDM compared to their counterparts without a history of GDM [10]. Women with GDM history are at high risk for recurrence of GDM and the development of type 2 diabetes, however, the general condition of their subsequent pregnancy remains unclear. Most articles have focused on postpartum metabolic characteristics of women with GDM history, but less attention has been given to the differences between women with previous GDM and the general pregnant women during a subsequent pregnancy. In this regard, our study attempted to elucidate different clinical characteristics, glucose metabolism, lipid metabolism, gestational weight gain (GWG) at various stages of pregnancy, and pregnancy outcomes of women with GDM history and those without GDM history. Meanwhile, we investigated GWG effect before oral glucose tolerance test (OGTT) on pregnancy outcomes of women with prior GDM, aiming to produce relevant information to guide proper management methods during pregnancy and reduce related adverse maternal and infant outcomes.

\section{Materials and methods \\ Study subjects}

We conducted a retrospective cohort study comprising pregnant women who established a medical record for receiving healthcare and delivered singleton gestations at Beijing Obstetrics and Gynecology Hospital affiliated to Capital Medical University from January 1, 2017 until December 31, 2018. On the one hand, participants were included in the study if they; (i) were multipara; (ii) were women who used to diagnosed with GDM after 2011. On the other hand, subjects were excluded if they; (i) were aged $>45$ or $<18$ years old; (ii) were females with multiple pregnancies; (iii) had pre-existing diabetes, hypertension, acute and chronic heart, liver, kidney disease, or other serious diseases; (iv) exhibited fetal chromosomal abnormalities or major birth defects and; (v) did not have complete clinical data. According to the above criteria, 441 pregnant women with a history of GDM were included as the study group. At the same time, the mechanical sampling methods was used to sort every 40 pregnant women with no history of GDM as a list according to the medical record number. The odd-numbered pregnant women were selected. Finally, 1637 women without GDM history treated in the same period were randomly recruited as the control group. The study received ethical approval from the ethics committee of Beijing Obstetrics and Gynecology Hospital affiliated with Capital Medical University(2018-KY-030-01).

\section{Study design}

All pregnant women with previous GDM underwent fasting plasma glucose (FPG) test at 6-8 weeks of gestation unless insulin or metformin treatment was initiated earlier. $\mathrm{FPG} \geq 7.0 \mathrm{mmol} / \mathrm{L}$ indicates pre-gestational diabetes mellitus. These women were managed according to the standard of pre-pregnancy diabetes. The rest of pregnant women with prior GDM underwent a 75-g OGTT at $24-28$ weeks of gestation, FPG $\geq 5.1 \mathrm{mmol} / \mathrm{L}, 1-\mathrm{h}$ value $\geq 10.0 \mathrm{mmol} / \mathrm{L}$, or 2 - $\mathrm{h}$ value $\geq 8.5 \mathrm{mmol} / \mathrm{L}$ indicate GDM. Height and weight were recorded without shoes at the outpatient clinic or subject's home. Pre-pregnancy BMI was calculated as weight divided by height squared $\left(\mathrm{kg} / \mathrm{m}^{2}\right)$ using self-reported pre-pregnancy weight. Each subject in the study group received individualized counseling on diet, physical activity and weight control from trained study nurses and doctors in the first visit (usually from 8-12 weeks of gestation). A follow-up was performed every 2-4 weeks included formulating diet prescription according to pre-pregnancy body mass index (BMI) and physical activities. The control group received standard antenatal care. We used the electronic medical record system of hospital to collect patient-level variables, such as standard demographic information (age, pre-pregnancy height and weight, GWG, number of prior pregnancies, parity, family history, previous history, history of pregnancy and childbirth and education level) and laboratory data (glucose and lipid metabolism indexes at various stages of pregnancy), as well as relevant maternal and infant outcomes. Then, the above indicators of the two groups were compared statistically. Meanwhile, in women with previous GDM, we discussed the influence of GDM history on the risk of GDM and pregnancy-induced hypertension (included gestational hypertension and pre-eclampsia). We also investigated the association between GDM, pregnancy-induced hypertension and GWG before OGTT in the study group. Maternal GWG before OGTT was used to group the subjects within or above the target as recommended by the Institute of Medicine (IOM). GWG below or above the recommended threshold was defined as inadequate or excessive weight gains, respectively. The risk of GDM and pregnancy-induced hypertension in different subgroups was analyzed.

\section{Laboratory test indicators and pregnancy outcome}

Blood lipid levels were determined at various stages of pregnancy using a full-automatic biochemical analyzer. The analysis included quantification of triglycerides (TG), total cholesterol (TC), high-density lipoprotein-cholesterol (HDL-C), and low-density lipoprotein-cholesterol (LDL-C) levels. Blood glucose levels were determined using the oxidase method during 
pregnancy. Gestational hypertension (GH) was defined as de novo hypertension if an individual exhibited a systolic blood pressure $\geq 140 \mathrm{mmHg}$ and, or diastolic blood pressure $\geq 90 \mathrm{mmHg}$ after 20 weeks of pregnancy [11], whereas pre-eclampsia (PE) was defined by the onset of hypertension and proteinuria after 20 weeks of gestation [12]. Postpartum hemorrhage (PPH) was defined as blood loss $\geq 500 \mathrm{~mL}$ within $24 \mathrm{~h}$ of delivery. Macrosomia was defined as a birth weight $\geq 4000 \mathrm{~g}$, whereas preterm birth was defined as gestational age $<37$ weeks. Polyhydramnios was defined as an amniotic fluid index $\geq 25 \mathrm{~cm}$, whereas oligohydramnios was defined as an amniotic fluid index $\leq 5 \mathrm{~cm}$. Small for gestational age (SGA) was defined as a birth weight below the 10th percentile for gestational age and gender, whereas large for gestational age (LGA) was defined as a birth weight above the 90th percentile for gestational age and gender. Lastly, premature rupture of membranes refers to a patient who is beyond 37 weeks of gestation and has presented with rupture of membranes before the onset of labor.

\section{Statistical analysis}

All data were evaluated using the SPSS 23.0 software. Normal distribution data are presented as mean \pm SD, and a t-test is utilized to compare groups. Non-normal distribution data are presented as median (25th-75th percentile) [M (P25-P75)], and the rank sum test is employed to compare between groups. Enumeration data is expressed by frequency and percentage, and the chisquare test is deployed to compare between groups. A value of $p<0.05$ was regarded as statistically significant.

\section{Description of covariates}

In 441 women with prior GDM, we performed multivariable logistic regression analysis to assess the influence of GDM history on GDM and pregnancy-induced hypertension after controlling for relevant confounding factors, such as maternal age, pre-pregnancy BMI, gravidity, parity, gestational age at delivery, cigarette smoke and alcohol consumption pre-pregnancy, family history of diabetes, glucose metabolism, lipid metabolism and GWG before OGTT. Furthermore, multivariable logistic regression analysis was applied to investigate the correlation between GDM, pregnancy-induced hypertension and GWG before OGTT of women with GDM history after controlling for relevant confounding factors, such as maternal age, pre-pregnancy BMI, gravidity, parity, gestational age at delivery, cigarette smoke pre-pregnancy and alcohol consumption pre-pregnancy.

\section{Results}

Women with previous GDM had a significantly higher maternal age $(34.8 \pm 3.5$ vs. $34.2 \pm 3.6$ years old, $\mathrm{p}=0.002)$ and higher pre-pregnancy BMI (23.3 \pm 3.5 vs. $\left.22.2 \pm 3.4 \mathrm{~kg} / \mathrm{m}^{2}, \mathrm{p}<0.001\right)$ compared with the control group. Parity ( $2.0 \pm 0.3$ vs. $2.0 \pm 0.2, \mathrm{p}<0.001)$, abnormal pregnancy history $(19.7 \%$ vs. $13.0 \%, \mathrm{p}<0.001), \mathrm{GH}$ history $(4.8 \%$ vs. $2.1 \%, \mathrm{p}<0.05)$, macrosomia history $(7.0 \%$ vs. $1.9 \%, \mathrm{p}<0.001)$, and PCOS history $(2.3 \%$ vs. $0.4 \%$, $\mathrm{p}<0.001)$ were significantly higher in those with prior GDM, relative to women without GDM history. Besides, a family history of type 2 diabetes was present in almost one-third of the study group participants compared to a tenth of the controls ( $31.3 \%$ vs. $10.8 \%, \mathrm{p}<0.001)$. Furthermore, women with GDM history had a higher education degree $(76.2 \%$ vs. $70.7 \%, \mathrm{p}<0.05)$. No significant differences were seen between the two groups regarding gravidity, smoking, and PE history ( $\mathrm{p}>0.05$, Table 1$)$.

In the 1st trimester of pregnancy, relative to women without GDM history, those with GDM history exhibited significantly higher FPG levels [4.9 (4.7-5.3) vs. 4.8 (4.5-5.0) $\mathrm{mmol} / \mathrm{L}, \mathrm{p}<0.001]$ and higher TG levels $[1.3$ $(0.9-1.7)$ vs. $1.1(0.8-1.5) \mathrm{mmol} / \mathrm{L}, \mathrm{p}<0.001]$. Moreover, in the 2nd trimester of pregnancy, women with previous GDM exhibited significantly higher FPG levels [ 4.8 (4.5-5.1) vs. $4.5(4.3-4.8) \mathrm{mmol} / \mathrm{L}, \mathrm{p}<0.001]$, higher TG levels [2.4 (1.9-3.0) vs. $2.3(1.8-2.9) \mathrm{mmol} / \mathrm{L}, \mathrm{p}<0.05]$, higher TC levels [6.1 (5.4-6.7) vs. $5.8(5.0-6.5) \mathrm{mmol} / \mathrm{L}$, $\mathrm{p}<0.001]$, as well as significantly higher LDL-C levels [3.1 (2.6-3.7) vs. $2.9(2.4-3.5) \mathrm{mmol} / \mathrm{L}, \mathrm{p}<0.001]$ compared with the control group. In the 3rd trimester of pregnancy, women prior GDM exhibited significantly higher FPG levels [4.6 (4.3-5.0) vs. $4.4(4.1-4.7) \mathrm{mmol} / \mathrm{L}, \mathrm{p}<0.001]$, higher TC levels [6.4 (5.7-7.2) vs. $6.1(5.3-7.0) \mathrm{mmol} / \mathrm{L}$, $\mathrm{p}<0.001]$, as well as significantly higher LDL-C levels [3.4 (2.7-4.0) vs. $3.2(2.5-3.8) \mathrm{mmol} / \mathrm{L}, \mathrm{p}<0.001$ ], relative to those without GDM history. Conversely, HDL-C levels throughout pregnancy were significantly lower in the study group $[1.5(1.2-1.7)$ vs. $1.6(1.4-1.9) \mathrm{mmol} / \mathrm{L}$, $1.8(1.5-1.9)$ vs. $1.9(1.7-2.2) \mathrm{mmol} / \mathrm{L}, 1.7(1.5-1.9)$ vs. $1.8(1.5-2.0) \mathrm{mmol} / \mathrm{L}, \mathrm{p}<0.001]$, relative to the control group. Nevertheless, TC and LDL-C levels in the 1st trimester of pregnancy, and TG levels in the 3rd trimester, were not significantly different $(\mathrm{p}>0.05$, Table 1$)$.

GWG before 16 weeks of gestation, GWG before OGTT, GWG between OGTT and delivery, and GWG during entire pregnancy were significantly lower in the study group $(2.0 \pm 2.5$ vs. $2.4 \pm 2.7 \mathrm{~kg}, 6.3 \pm 3.3$ vs. $7.9 \pm 3.7 \mathrm{~kg}, 3.6 \pm 2.1$ vs. $4.0 \pm 2.1 \mathrm{~kg}, 11.8 \pm 4.6$ vs. $14.4 \pm 4.6 \mathrm{~kg}, \mathrm{p}<0.05]$ relative to the control group. On the contrary, significantly higher incidence of GDM $(46.5 \%$ vs. $18.1 \%, \mathrm{p}<0.001)$ and pregnancy-induced hypertension $(8.4 \%$ vs. $5.4 \%, \mathrm{p}<0.05)$ were observed in 
Table 1 Clinical characteristics, glucose and lipid indexes and pregnancy oitcomes of women with and without GDM history

\begin{tabular}{|c|c|c|c|}
\hline & $\begin{array}{l}\text { Study group } \\
(n=441)\end{array}$ & $\begin{array}{l}\text { Control group } \\
(n=1637)\end{array}$ & P-value \\
\hline Age, years & $34.8 \pm 3.5$ & $34.2 \pm 3.6$ & 0.002 \\
\hline Pre-pregnancy BMl, kg/m² & $23.3 \pm 3.5$ & $22.2 \pm 3.4$ & $<0.001$ \\
\hline Gravidity, \% & $2.9 \pm 1.1$ & $2.9 \pm 1.0$ & 0.5 \\
\hline Parity, \% & $2.0 \pm 0.3$ & $2.0 \pm 0.2$ & 0.001 \\
\hline Family history of diabetes, $\%$ & $138(31.3)$ & $176(10.8)$ & $<0.001$ \\
\hline Histories of abnormal pregnancy, \% & $87(19.7)$ & $213(13.0)$ & $<0.001$ \\
\hline History of GH, \% & $21(4.8)$ & $34(2.1)$ & 0.002 \\
\hline History of PE, \% & $3(0.7)$ & $8(0.5)$ & 0.6 \\
\hline History of macrosomia, \% & $31(7.0)$ & $31(1.9)$ & $<0.001$ \\
\hline History of PCOS, $\%$ & $10(2.3)$ & $6(0.4)$ & $<0.001$ \\
\hline Smoking, \% & $25(5.7)$ & $98(6.0)$ & 0.8 \\
\hline \multicolumn{4}{|l|}{ Education, \% } \\
\hline Undergraduate and above & $336(76.2)$ & $1157(70.7)$ & \multirow[t]{2}{*}{0.02} \\
\hline Junior college and below & $105(23.8)$ & $480(29.3)$ & \\
\hline \multicolumn{4}{|c|}{ Glucose and lipid profiles in the first trimester, $\mathrm{mmol} / \mathrm{L}$} \\
\hline $\mathrm{TC}$ & $4.2(3.8-4.8)$ & $4.3(3.8-4.8)$ & 0.6 \\
\hline TG & $1.3(0.9-1.7)$ & $1.1(0.8-1.5)$ & $<0.001$ \\
\hline $\mathrm{HDL}-\mathrm{C}$ & $1.5(1.2-1.7)$ & $1.6(1.4-1.9)$ & $<0.001$ \\
\hline LDL-C & $2.2(1.8-2.6)$ & $2.1(1.8-2.6)$ & 0.1 \\
\hline FPG & $4.9(4.7-5.3)$ & $4.8(4.5-5.0)$ & $<0.001$ \\
\hline \multicolumn{4}{|c|}{ Glucose and lipid profiles in the second trimester, $\mathrm{mmol} / \mathrm{L}$} \\
\hline $\mathrm{TC}$ & $6.1(5.4-6.7)$ & $5.8(5.0-6.5)$ & $<0.001$ \\
\hline TG & $2.4(1.9-3.0)$ & $2.3(1.8-2.9)$ & 0.04 \\
\hline $\mathrm{HDL}-\mathrm{C}$ & $1.8(1.5-1.9)$ & $1.9(1.7-2.2)$ & $<0.001$ \\
\hline LDL-C & $3.1(2.6-3.7)$ & $2.9(2.4-3.5)$ & $<0.001$ \\
\hline OGTT fasting glucose & $4.8(4.5-5.1)$ & $4.5(4.3-4.8)$ & $<0.001$ \\
\hline OGTT 1-h glucose & $9.1(7.9-10.2)$ & $7.5(6.5-8.6)$ & $<0.001$ \\
\hline OGTT 2-h glucose & $7.5(6.6-8.5)$ & $6.4(5.6-7.3)$ & $<0.001$ \\
\hline \multicolumn{4}{|c|}{ Glucose and lipid profiles in the third trimester, $\mathrm{mmol} / \mathrm{L}$} \\
\hline $\mathrm{TC}$ & $6.4(5.7-7.2)$ & $6.1(5.3-7.0)$ & $<0.001$ \\
\hline TG & $3.1(2.5-3.8)$ & $3.0(2.4-3.7)$ & 0.2 \\
\hline $\mathrm{HDL}-\mathrm{C}$ & $1.7(1.5-1.9)$ & $1.8(1.5-2.0)$ & 0.001 \\
\hline LDL-C & $3.4(2.7-4.0)$ & $3.2(2.5-3.8)$ & $<0.001$ \\
\hline FPG & $4.6(4.3-5.0)$ & $4.4(4.1-4.7)$ & $<0.001$ \\
\hline GWG before 16 weeks of gestation, kg & $2.0 \pm 2.5$ & $2.4 \pm 2.7$ & 0.02 \\
\hline GWG before OGTT, kg & $6.3 \pm 3.3$ & $7.9 \pm 3.7$ & $<0.001$ \\
\hline GWG between OGTT and delivery, kg & $3.6 \pm 2.1$ & $4.0 \pm 2.1$ & $<0.001$ \\
\hline GWG during the entire pregnancy, kg & $11.8 \pm 4.6$ & $14.4 \pm 4.6$ & $<0.001$ \\
\hline Gestational week at delivery, weeks & $38.2 \pm 1.6$ & $38.4 \pm 1.5$ & 0.02 \\
\hline \multicolumn{4}{|l|}{ Delivery mode, $n$ (\%) } \\
\hline Vaginal or assisted vaginal delivery & $224(50.8)$ & $824(50.3)$ & \multirow[t]{2}{*}{0.9} \\
\hline Caesarean section & $217(49.2)$ & $813(49.7)$ & \\
\hline $\mathrm{GH}$ and $\mathrm{PE}, \mathrm{n}(\%)$ & $37(8.4)$ & $88(5.4)$ & 0.02 \\
\hline GDM, n (\%) & $205(46.5)$ & $296(18.1)$ & $<0.001$ \\
\hline $\mathrm{PPH}, \mathrm{n}(\%)$ & $67(15.2)$ & $293(17.9)$ & 0.2 \\
\hline Macrosomia, n (\%) & $46(10.4)$ & $148(9.0)$ & 0.4 \\
\hline Premature rupture of membrane, n (\%) & $84(19.1)$ & $275(16.8)$ & 0.3 \\
\hline Polyhydramnios and oligohydramnios, n (\%) & $18(4.1)$ & $49(3.0)$ & 0.3 \\
\hline
\end{tabular}


Table 1 (continued)

\begin{tabular}{llll}
\hline & $\begin{array}{l}\text { Study group } \\
(\mathbf{n = 4 4 1 )}\end{array}$ & $\begin{array}{l}\text { Control group } \\
(\mathbf{n = 1 6 3 7 )}\end{array}$ & P-value \\
\hline SGA, n (\%) & $11(2.5)$ & $54(3.3)$ & 0.4 \\
LGA, n (\%) & $57(12.9)$ & $158(9.7)$ & 0.04 \\
Preterm birth, $\mathrm{n}(\%)$ & $34(7.7)$ & $94(5.7)$ & 0.1 \\
Birth weight, g & $3386 \pm 548$ & $3388 \pm 504$ & 0.7 \\
\hline
\end{tabular}

Table 2 The relationship between pregnancy outcomes and GDM history

\begin{tabular}{lll}
\hline & Without GDM history & $\begin{array}{l}\text { With GDM history } \\
\text { Adjusted OR } \mathbf{9 5 \%} \text { Cl }\end{array}$ \\
\cline { 2 - 3 } & $\mathbf{( N = 1 6 3 7 )}$ & $\mathbf{( N = 4 4 1 )}$ \\
\hline GDM & 1 & $3.25[2.26-4.68]^{* *}$ \\
GH and PE & 1 & $1.50[1.05-2.45]^{*}$ \\
LGA & 1 & $1.34[0.96-1.87]$ \\
\hline
\end{tabular}

Data was analyzed using multivariable logistic regression analysis. Models were adjusted for maternal age, pre-pregnancy BMI, gravidity, parity, gestational age at delivery, cigarette smoke pre-pregnancy, alcohol consumption prepregnancy, family history of diabetes, glucose and lipid metabolism and GWG in the 1 st and 2 nd trimesters

Reference group: women without GDM history

$O R$ odds ratio, $\mathrm{Cl}$ confidence interval

${ }^{*} \mathrm{p}<0.05$

** $p<0.005$

the study group relative to the control group. The multivariate logistic regression results demonstrated that women with prior GDM had a higher risk of developing GDM (OR 3.25, 95\% CI 2.26-4.68) and pregnancyinduced hypertension (OR 1.50, 95\% CI 1.05-2.45) compared with those without previous GDM. Besides, relative to women without previous GDM, the LGA rate was higher in those with GDM history (12.9\% vs. 9.7\%, $\mathrm{p}<0.05)$. On the contrary, the gestational week at delivery was lower in those with GDM history (38.2 \pm 1.6 vs. $38.4 \pm 1.5$ weeks, $\mathrm{p}<0.05)$. Delivery mode, $\mathrm{PPH}$, macrosomia, premature membrane rupture, polyhydramnios and oligohydramnios, SGA, preterm birth, and birth weight were not significantly different $(\mathrm{p}>0.05$, Table 1 , 2).

Table 3 shows the relationship between GDM, pregnancy-induced hypertension and GWG before OGTT in women with GDM history. Multivariate logistic regression demonstrated that compared with women in the inadequate GWG group, individuals in the excessive GWG group had a higher risk of developing pregnancyinduced hypertension (OR 1.47, 95\% CI 1.05-3.32), while inadequate GWG was not a protective factor for GDM and pregnancy-induced hypertension (Table 3 ).
Table 3 The relationship between GDM, pregnancy-induced hypertension and GWG before OGTT in women with GDM history

\begin{tabular}{lcc}
\hline GWG before OGTT & $\begin{array}{c}\text { Inadequate }(\mathbf{N}=122) \\
\text { Adjusted OR 95\% Cl }\end{array}$ & $\begin{array}{c}\text { Excessive }(\mathbf{N}=119) \\
\text { Adjusted OR 95\% Cl }\end{array}$ \\
\hline GDM & $0.93[0.56-1.55]$ & $1.08[0.65-1.81]$ \\
GH and PE & $0.34[0.11-1.11]$ & $1.47[1.05-3.32]^{*}$ \\
\hline
\end{tabular}

Data was analyzed using multivariable logistic regression analysis. Models were adjusted for maternal age, pre-pregnancy BMI, gravidity, parity, gestational age at delivery, cigarette smoke pre-pregnancy and alcohol consumption prepregnancy

Reference group: adequate GWG group in women with GDM history

$O R$ odds ratio, $\mathrm{Cl}$ confidence interval

${ }^{*} p<0.05$

** $p<0.005$

\section{Discussion}

Our data suggested that FPG during the entire pregnancy, TG levels in the 1st and 2nd trimesters, TC and LDL-C levels in the 2nd and 3rd trimesters in women with previous GDM were higher than those without GDM history. Inversely, relative to the control group, women with GDM history exhibited significantly lower HDL-C levels during the whole pregnancy. Intriguingly, although GWG at various pregnancy stages were lower in women with previous GDM than those without GDM history, we still found a higher risk of GDM and pregnancy-induced hypertension in women with prior GDM. Furthermore, in women with GDM history, excessive GWG increased the risk of developing pregnancyinduced hypertension, while insufficient GWG had no protective effect on the occurrence of GDM and pregnancy-induced hypertension.

The high risk of type 2 diabetes and recurrence in subsequent pregnancies has been well documented in women with GDM history $[13,14]$, and the recurrence rate may vary according to ethnic differences and characteristics of participants $(30-84 \%)[15,16]$. Prior studies found a high risk of type 2 diabetes and abnormal glucose tolerance in women with previous GDM, with an incidence of up to four times greater than general women [17]. Consistent with previous studies, our data indicated 
that women with previous GDM had a threefold risk for GDM $(\mathrm{OR}=3.25$, 95\% CI 2.26-4.68) relative to counterparts without GDM history. Our study also demonstrated that GDM history is one of the risk factors of GH and $\mathrm{PE}$. In particular, the risk of developing $\mathrm{GH}$ and $\mathrm{PE}$ in women with prior GDM is $1.50(\mathrm{OR}=1.50$, 95\% CI 1.05$2.45)$ times higher than the control group. Mounting evidence suggested that women with GDM history are at higher risk of metabolic syndrome and cardiovascular disease, including hypertension, dyslipidemia, and obesity [18-20]. Hromadnikova et al. [21] uncovered longterm epigenetic changes in mothers' peripheral blood with GDM history that may predispose them to diabetes, cardiovascular, and cerebrovascular diseases. The dyslipidemia characteristics in our paper may be one reason for the high incidence of pregnancy-induced hypertension in women with a history of GDM.

Persistent high insulin resistance during pregnancy can stress and exacerbate pancreatic cells' damage [22, 23]. Multiple studies revealed that women with GDM had varying insulin resistance levels and reduced insulin sensitivity after delivery [24-27]. A Greek study found that women with previous GDM presented various degrees of impaired fasting glucose and impaired glucose tolerance in postpartum [28]. Herein, we did not measure insulin levels; however, our data illustrated that women with previous GDM exhibited significantly higher FPG in early pregnancy stages than the control group. It was suggested that abnormal glucose metabolism in GDM may not end with delivery but persist postpartum and carry the condition into subsequent pregnancies. Meanwhile, our study also indicated that abnormal lipid metabolism exists at the early stage of pregnancy in the study group, including higher TG, TC, LDL-C, and lower HDL-C levels. Similarly, numerous studies also confirmed that dyslipidemia increases the risk of GDM $[29,30]$. This may be attributed to the following two reasons. Excess TG was stored in skeletal muscles, which may impair insulin signaling, and eventually, the lipotoxic effect of FFAs leads to decreased insulin-stimulated skeletal muscle glucose transport [31]. In addition, oxidative stress, secondary to dyslipidemia, may ultimately cause decreased insulin gene expression and insulin secretion impairment [32].

Excessive GWG is a well-established risk factor for GDM [33-36]. We evaluated GWG at various pregnancy stages in two groups of women. Our findings suggested that GWG before 16 weeks and GWG before OGTT were significantly lower in women with GDM history than those without GDM history. Consistent with our results, Persson et al. [37] also found that women with GDM history gained less GWG than those without GDM history, related to the extensive intervention of GWG initiated from early pregnancy. However, they did not analyze GWG in various pregnancy stages, which is discussed in our article for the first time. In our hospital, women with previous GDM were provided individualized medical nutritional therapy (MNT) and exercise guidance to help control GWG in the first visit (usually from 8-12 weeks of gestation). To prevent GDM recurrence in these women, a follow-up was performed every 2-4 weeks, including formulating diet prescription according to BMI and physical activities. Once GDM was diagnosed, in addition to MNT, more stringent weight gain targets were set, and insulin therapy was given when necessary. From our data, our intervention resulted in significantly lower GWG before 16th weeks of gestation $(0.4 \mathrm{~kg}$ less than the control group in this study) and before OGTT (1.6 kg less than the control group in this study) in women with previous GDM than those without GDM history. Nevertheless, in the present study, GDM incidence in women with GDM history is still much higher than in the control group. Comparably and of particular note, our findings further indicated that excessive GWG before OGTT exhibited a significantly positive correlation with pregnancy-induced hypertension in women with previous GDM, compared with GWG within the IOM guidelines in women with GDM history. Nevertheless, inadequate GWG before OGTT did not have a protective effect on GDM and pregnancyinduced hypertension. Our finding was inconsistent with previous studies involving general pregnant women [38]. Whether the complicated endocrine and metabolism in women with GDM history are involved should be further investigated. Besides, this seems to suggest that a limited reduction of GWG before OGTT may not be enough to offset the effects of pre-existing impaired glucose and lipid metabolism on adverse pregnancy outcomes in women with GDM history. However, the superposition effect of GDM history and excessive GWG state may contribute to increasing pregnancy-induced hypertension. Similar to our findings, a multicenter randomized controlled trial in Europe indicated that lifestyle change, even with an average $4.3 \mathrm{~kg}$ GWG reduction in the combined diet and exercise interventions by $35-37$ weeks, is insufficient to prevent GDM in overweight/obese women [39]. Whether a larger amount of GWG reduction before OGTT or initiation of intervention before conception may result in a decline in GDM in women with GDM history remains unknown, which urgently needs to be explored in the future.

\section{Strength and limitation}

The strength of this study lies in having a larger sample size, which fully integrated data on glucose and lipid metabolism, and GWG at various pregnancy stages for both groups. Furthermore, for the first time, we 
conducted a subgroup analysis of GWG extent in women with GDM history and found that even a $1.6 \mathrm{~kg}$ reduction in GWG before OGTT did not reduce the risk of GDM and pregnancy-induced hypertension. However, our study has limitations. This study was not a randomized controlled trial but a retrospective analysis. Moreover, the study was limited to single-center data, indicating that our findings may not reflect individuals from other Chinese regions or countries, owing to potential differences in education and socioeconomic levels. The current data were also insufficient to answer definitively essential questions of how much reduction of GWG or when to intervene can improve the adverse outcomes.

\section{Conclusion}

In summary, for women with GDM history, the superposition effect of advanced age, higher pre-pregnancy BMI, as well as glucose and lipid metabolism disorders in the 1st trimester can lead to adverse pregnancy outcomes. Limited reduction of GWG may not be enough to reduce the incidence of adverse pregnancy outcomes in those with GDM history. Relevant and necessary management may need to be initiated at early stage or even before pregnancy. Furthermore, it is unclear how much GWG during pregnancy and what kind of patterns of GWG are applicable to women with GDM history. Therefore, large sample-sized and multicenter studies need to confirm when and what kind of intervention intensity is appropriate for women with previous GDM.

\section{Abbreviations \\ GDM: Gestational diabetes mellitus; GWG: Gestational weight gain; OGTT: Oral glucose tolerance test; FPG: Fasting plasma glucose; BMI: Body mass index; IOM: Institute of Medicine; TG: Triglycerides; TC: Total cholesterol; HDL-C: High density lipoprotein-cholesterol; LDL-C: Low density lipoprotein-cholesterol; GH: Gestational hypertension; PE: Preeclampsia; PPH: Postpartum hemorrhage; SGA: Small for gestational age; LGA: Large for gestational age; OR: Odds ratio;} Cl: Confidence interval.

\section{Acknowledgements}

The authors thank the participants for participating in the study and the medical staff for their work on information collection.

\section{Authors' contributions}

All the authors contributed significantly to the manuscript. XL was primarily responsible for the data analysis and writing of the manuscript. WZ significantly revised the draft, interpreted the data, and involved in data analyses. $C L$ and LRZ collected the information and participated in data interpretation. $\mathrm{LZ}$ and ZHT involved in the data management and draft revision. GHL was responsible for designing the study and critically revising the manuscript. All authors read and approved the final manuscript.

\section{Funding}

This study was supported by Capital's Funds for Health Improvement and Research (2018-2-2112); National Key Research and Development Program of China (2016YFC1000304); Beijing Hospitals Authority' Ascent Plan (DFL20191402).

\section{Availability of data and materials}

The data is available upon reasonable request to the corresponding author.

\section{Declarations}

\section{Ethics approval and consent to participate}

The study was approved by the Ethics Committee of Beijing Obstetrics and Gynecology Hospital (2012-KY-012, 2016-KY-066). Written informed consent was obtained from all participants. All procedures were performed in compliance with the Declaration of Helsinki.

\section{Consent for publication}

Not applicable.

\section{Competing interests}

The authors declare that they have no competing interests.

Received: 18 March 2021 Accepted: 24 June 2021

Published online: 06 July 2021

\section{References}

1. Wendland EM, Torloni MR, Falavigna M, et al. Gestational diabetes and pregnancy outcomes-a systematic review of the World Health Organization (WHO) and the International Association of Diabetes in Pregnancy study groups (IADPSG) diagnostic criteria. BMC Pregnancy Childbirth. 2012;12:23

2. Bellamy L, Casas J-P, Hingorani AD, Williams D. Type 2 diabetes mellitus after gestational diabetes: a systematic review and meta-analysis. Lancet. 2009:373:1773-9.

3. Metzger BE, Coustan DR, Trimble ER. Hyperglycemia and adverse pregnancy outcomes. Clin Chem. 2019;65(7):937-8.

4. Yumei W, Huixia Y. Development and Prospect of gestational diabetes research in China. Chin J Perinatal Med. 2018;21(4):218-20. https://doi. org/10.3760/cma.j.issn.1007-9408.2018.04.002.

5. Liu J, Song L, Qiu J, et al. Reducing maternal mortality in China in the era of the two-child policy. BMJ Glob Health. 2020;5(2):e002157.

6. Teng $X$, Shane MI, Pan S. The changing situation about maternal age, risk factors and pregnancy outcomes after the two-child policy: a retrospective cohort study. Ann Palliat Med. 2020;9(3):824-34.

7. Li Z, Cheng Y, Wang D, et al. Incidence rate of type 2 diabetes mellitus after gestational diabetes mellitus: a systematic review and meta-analysis of 170,139 women. J Diabetes Res. 2020;27(2020):3076463.

8. Bellamy L, Casas JP, Hingorani AD, et al. Type 2 diabetes mellitus after gestational diabetes: a systematic review and meta-analysis. Lancet. 2009;373:1773-9.

9. Vounzoulaki E, Khunti K, Abner SC, et al. Progression to type 2 diabetes in women with a known history of gestational diabetes: systematic review and meta-analysis. BMJ. 2020;369:m1361.

10. Shin D, Lee KW. High pre-pregnancy BMI with a history of gestational diabetes mellitus is associated with an increased risk of type 2 diabetes in Korean women. PLoS ONE. 2021;16(6):e0252442. https://doi.org/10.1371/ journal.pone.0252442 (eCollection2021).

11. Bernard N, Forest JC, Tarabulsy GM, Bujold E, Bouvier D, Giguere Y. Use of antidepressants and anxiolytics in early pregnancy and the risk of preeclampsia and gestational hypertension: a prospective study. BMC Pregnancy Childbirth. 2019;19(1):146.

12. Williamson RD, McCarthy FP, Kenny LC, McCarthy CM. Activation of a TLR9 mediated innate immune response in preeclampsia. Sci Rep. 2019;9(1):5920

13. Tieu J, Shepherd E, Middleton P, et al. Interconception care for women with a history of gestational diabetes for improving maternal and infant outcomes. Cochrane Database Syst Rev. 2017:8(8):CD010211.

14. Kim C, Berger DK, Chamany S. Recurrence of gestational diabetes mellitus: a systematic review. Diabetes Care. 2007;30(5):1314-9.

15. England $\mathrm{L}$, Kotelchuck $\mathrm{M}$, Wilson $\mathrm{HG}$, et al. Estimating the recurrence rate of gestational diabetes mellitus (GDM) in Massachusetts 1998-2007: methods and findings. Matern Child Health J. 2015;19(10):2303-13.

16. Ajala $O$, Jensen LA, Ryan E, Chik C. Women with a history of gestational diabetes on long-term follow up have normal vascular function despite more dysglycemia, dyslipidemia and adiposity. Diabetes Res Clin Pract. 2015:110(3):309-14. 
17. Fan Y, Li W, Liu H, et al. Effects of obesity and a history of gestational diabetes on the risk of postpartum diabetes and hyperglycemia in Chinese women: obesity, GDM and diabetes risk. Diabetes Res Clin Pract. 2019;156:107828.

18. Reece EA, Leguizamon G, Wiznitzer A. Gestational diabetes: the need for a common ground. Lancet. 2009;373(9677):1789-97.

19. Reece EA. The fetal and maternal consequences of gestational diabetes mellitus. J Maternal Fetal Neonatal Med. 2010;23(3):199-203.

20. Kessous R, Shoham-Vardi I, Pariente G, et al. An association between gestational diabetes mellitus and long-term maternal cardiovascular morbidity. Heart. 2013;99:1118-21.

21. Hromadnikova I, Kotlabova K, Dvorakova L, et al. Diabetes mellitus and cardiovascular risk assessment in mothers with a history of gestational diabetes mellitus based on postpartal expression profile of MicroRNAs associated with diabetes mellitus and cardiovascular and cerebrovascular diseases. Int J Mol Sci. 2020;21:7.

22. Vambergue A, Dognin C, Boulogne A, et al. Increasing incidence of abnormal glucose tolerance in women with prior abnormal glucose tolerance during pregnancy: DIAGEST 2 study. Diabet Med. 2008;25(1):58-64.

23. Nohira T, Kim S, Nakai H, et al. Recurrence of gestational diabetes mellitus: rates and risk factors from initial GDM and one abnormal GTT value. Diabetes Res Clin Pract. 2006;71(1):75-81.

24. Jin WY, Lin SL, Hou RL, et al. Associations between maternal lipid profile and pregnancy complications and perinatal outcomes: a populationbased study from China. BMC Pregnancy Childbirth. 2016;16:60.

25. Lim S, Versace VL, O'Reilly S, et al. Weight change and cardiometabolic outcomes in postpartum women with history of gestational diabetes. Nutrients. 2019;11:4

26. Molęda P, Homa K, Safranow K, et al. Women with normal glucose tolerance and a history of gestational diabetes show significant impairment of $\beta$-cell function at normal insulin sensitivity. Diabetes Metab. 2013:39(2):155-62.

27. Verma A, Boney CM, Tucker $\mathrm{R}$, et al. Insulin resistance syndrome in women with prior history of gestational diabetes mellitus. J Clin Endocrinol Metab. 2002;87(07):3227-35.

28. Apostolakis M, Paschou SA, Zapanti E, et al. HbA1c presents low sensitivity as a post-pregnancy screening test for both diabetes and prediabetes in Greek women with history of gestational diabetes mellitus. Hormones (Athens). 2018;17(2):255-9.

29. Ryckman KK, Spracklen CN, Smith CJ, et al. Maternal lipid levels during pregnancy and gestational diabetes: a systematic review and metaanalysis. BJOG. 2015;122(5):643-51.
30. Herrera E, Ortega-Senovilla H. Disturbances in lipid metabolism in diabetic pregnancy-Are these the cause of the problem? Best Pract Res Clin Endocrinol Metab. 2010;24:515-25.

31. Kelley DE, Goodpaster BH. Skeletal muscle triglyceride. An aspect of regional adiposity and insulin resistance. Diabetes Care. 2001;24(5):933-41.

32. Kajimoto $Y$, Kaneto $\mathrm{H}$. Role of oxidative stress in pancreatic betacell dysfunction. Ann N Y Acad Sci. 2004;1011(4):168-76.

33. Wang $C$, Wei $Y$, Zhang $X$, et al. A randomized clinical trial of exercise during pregnancy to prevent gestational diabetes mellitus and improve pregnancy outcome in overweight and obese pregnant women. Am J Obstet Gynecol. 2017;216(4):340-51.

34. Hashim M, Radwan H, Hasan H, et al. Gestational weight gain and gestational diabetes among Emirati and Arab women in the United Arab Emirates: results from the MISC cohort. BMC Pregnancy Childbirth. 2019;19:463.

35. Bouvier D, Forest J-C, Dion-Buteau E, et al. Association of maternal weight and gestational weight gain with maternal and neonate outcomes: a prospective cohort study. J Clin Med. 2019;8(12):2074.

36. Arora P, Tamber AB. Gestational weight gain among healthy pregnant women from Asia in comparison with institute of medicine (IOM) guidelines-2009: a systematic review. J Pregnancy. 2019;2019:3849596.

37. Persson M, Winkvist A, Mogren I. Lifestyle and health status in a sample of Swedish women four years after pregnancy: a comparison of women with a history of normal pregnancy and women with a history of gestational diabetes mellitus. BMC Pregnancy Childbirth. 2015;15:57.

38. Li C, Liu Y, Zhang W. Joint and independent associations of gestational weight gain and pre-pregnancy body mass index with outcomes of pregnancy in Chinese women: a retrospective cohort study. PLoS ONE. 2015;10(8):e0136850.

39. Simmons $D$, Devlieger $R$, van Assche A, et al. Association between gestational weight gain, gestational diabetes risk, and obstetric outcomes: a randomized controlled trial post hoc analysis. Nutrients. 2018;10(11):1568.

\section{Publisher's Note}

Springer Nature remains neutral with regard to jurisdictional claims in published maps and institutional affiliations.
Ready to submit your research? Choose BMC and benefit from:

- fast, convenient online submission

- thorough peer review by experienced researchers in your field

- rapid publication on acceptance

- support for research data, including large and complex data types

- gold Open Access which fosters wider collaboration and increased citations

- maximum visibility for your research: over $100 \mathrm{M}$ website views per year

At BMC, research is always in progress.

Learn more biomedcentral.com/submissions 